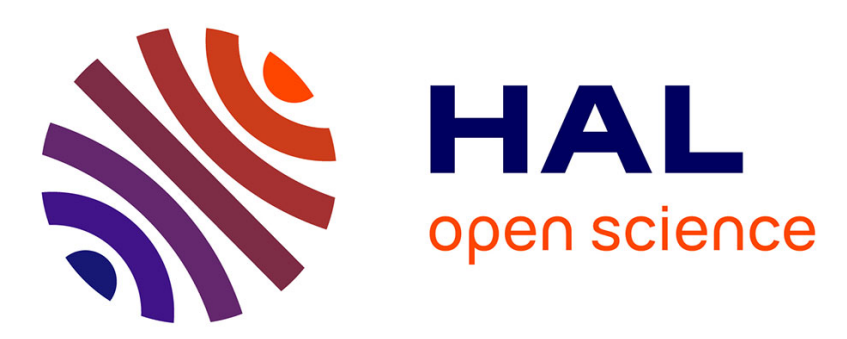

\title{
Manufacturing complexity evaluation at the design stage for both machining and layered manufacturing
}

\author{
Olivier Kerbrat, Pascal Mognol, Jean-Yves Hascoët
}

\section{To cite this version:}

Olivier Kerbrat, Pascal Mognol, Jean-Yves Hascoët. Manufacturing complexity evaluation at the design stage for both machining and layered manufacturing. CIRP Journal of Manufacturing Science and Technology, 2010, pp.208-215. 10.1016/j.cirpj.2010.03.007 . hal-00522092

\section{HAL Id: hal-00522092 \\ https://hal.science/hal-00522092}

Submitted on 29 Sep 2010

HAL is a multi-disciplinary open access archive for the deposit and dissemination of scientific research documents, whether they are published or not. The documents may come from teaching and research institutions in France or abroad, or from public or private research centers.
L'archive ouverte pluridisciplinaire HAL, est destinée au dépôt et à la diffusion de documents scientifiques de niveau recherche, publiés ou non, émanant des établissements d'enseignement et de recherche français ou étrangers, des laboratoires publics ou privés. 


\title{
MANUFACTURING COMPLEXITY EVALUATION AT THE DESIGN STAGE FOR BOTH MACHINING AND LAYERED MANUFACTURING
}

\author{
Authors: Olivier Kerbrat, Pascal Mognol, Jean-Yves Hascoët \\ IRCCyN (Institut de Recherche en Communications et Cybernétique de Nantes) \\ 1 rue de la Nö̈-BP 92101 - F-44321 Nantes Cedex 03, Nantes, France \\ Corresponding author: Olivier Kerbrat \\ IRCCyN / MO2P team \\ 1 rue de la Noë \\ BP 92101 \\ F-44321 Nantes Cedex 03 \\ France \\ Tel.: +33(0)299055275 \\ Fax: +33(0)299059328 \\ E-mail address: Olivier.Kerbrat@irccyn.ec-nantes.fr
}

\begin{abstract}
:
In this paper, a methodology to estimate manufacturing complexity for both machining and layered manufacturing is proposed in order to realize tools (dies or molds) by a combination of a subtractive and an additive process. Manufacturability indexes are calculated at the tool design stage, these indexes provide an accurate view of which areas of the tool will advantageously be machined or manufactured by an additive process. In this case, tools are not seen as single pieces but as 3-D puzzles with modules, manufactured aside by the best process and further assembled.
\end{abstract}

Keywords:

Design method, machining, layered manufacturing, octree 


\section{Introduction}

\subsection{Context of the study}

In order to stay competitive, manufacturers have to develop new products in a very short time and with reduced costs, whereas customers require more and more quality and flexibility. These objectives imply two design and manufacturing constraints: a rapid manufacturing and a high level of reactivity when design evolutions are required. The field of tooling (dies and molds) does not break these constraints and one possibility to improve competitiveness is to design and manufacture tools with modular and hybrid points of views.

\subsection{Modular point of view}

Instead of a one-piece tool, it is seen as a 3-D puzzle with modules realized separately and further assembled. The two advantages are: every module may be produced simultaneously and few modules may be changed without changing the whole tool. As it can be seen in the example in Fig. 1, the two alternatives of the product may be advantageously manufactured with the same mold, changing one module corresponding to the product model.

Fig. 1. Example of a modular die for two alternatives of a product.

\subsection{Hybrid point of view}

In the hybrid point of view, tools are decomposed into modules which will be manufactured by the best process, in term of time, cost and quality. The aim of this approach is to choose the best manufacturing process for each area of the tool. Presently, focus is put on comparison between a subtractive process (High-Speed Machining) and an additive process (Selective Laser Sintering). Nowadays, a number of additive fabrication technologies are used to produce metal parts and tools. These technologies provide an interesting alternative to CNC machining, especially in quickly manufacturing complex shapes, like conformal cooling channel or difficult-to-machine parts (which previously needed to be manufacture by EDM).

\subsection{Hybrid modular tooling}

The two points of view, hybrid and modular, have allowed creating rapid tools and rapid prototypes with the Multi Component Prototype concept [1]. This concept aims to decompose a mechanical prototype part on an assembly of several components. There are two main reasons for the multi-component decomposition:

To include the evolutionary requirement of the future parts to produce;

- $\quad$ To help designers to choose the best manufacturing process for each component, taking into account time, cost and feasibility of different fabrication technologies.

This paper is focused on the second point. The major problem is how to obtain a well-detailed view of the tool manufacturing complexity at the design stage in order to create a hybrid modular tool with a reduced complexity (and consequently with a lower manufacturing cost). This consideration forces the development of a manufacturability analysis with a well-detailed point of view. Manufacturability must be evaluated for the whole tool to discriminate which areas are the most complex to realize. This manufacturing complexity analysis is the main point of the tool design and manufacturing methodology presented in the next section.

\section{Hybrid modular tool design method}

The aim of this new methodology is to propose a decomposition of the tool which facilitates its manufacturing. So two points have to be taken into account: the evaluation of the manufacturability of the tool, and a hybrid modular decomposition that can improve the manufacturability. This method is divided into 6 stages, a schematic view is proposed in Fig. 2.

Fig. 2. Hybrid modular tool design methodology.

The starting point of this methodology is the one-piece tool CAD model. The manufacturing complexity of this one-piece tool is evaluated with the help of the manufacturability indexes calculation, defined in the next section. Then the modular and hybrid points of view are taken into account in order to create a hybrid modular tool. The 
manufacturability analysis is performed on this new tool CAD model. The last stage is a comparison between the two manufacturability analyses to quantify the advantages of the hybrid modular design.

This methodology has been implemented on a CAD software (SolidWorks 2007) with Visual Basic language [2] The section 3 presents the manufacturability analysis for both machining and layered manufacturing.

\section{Manufacturing complexity evaluation}

\subsection{Related works}

Many works have been done on manufacturability analysis, especially in the late 1990s, with the Design For Manufacturing (DFM) approach. DFM involves simultaneously considering design goals and manufacturing constraints in order to identify and to alleviate manufacturing problems while the product is being designed; thereby reducing the lead time for product development and improving product quality [3]. Most of the studies on DFM methods imply using a feature decomposition of the part CAD model, and associating manufacturability evaluation with each feature. The major problem is that features usually rely on one specific field. As an example, machining features are developed for CNC machining [4], but manufacturing features for additive technologies are still under development [5]. Furthermore, for free-form surface, usually used in tooling design, features do not bring enough information on the shape. So the hybrid modular tool manufacturability analysis cannot be based on feature decomposition, another tool CAD model decomposition method has to be found.

Each solid modeling method (CSG, B-rep ...) has its advantages and disadvantages relative to the others in term of accuracy, robustness, data structure and computing time. The key points are on the representation of parts with irregular surfaces, and approximation of curved surface.

Because there are often few geometric details of the tool that can change the manufacturing process choice (a small curvature radius of a concave shape for example); no information should be lost in the tool CAD model decomposition. And the decomposition accuracy must be at a high level for the areas that are geometrically complex (with lots of changes in surface orientations), whereas it may be lower for quite simple areas (a plane for instance). So octree decomposition [6] seems to be a good candidate for the tool CAD model decomposition.

\subsection{Octree concept}

An octree is a tree data structure, which represents a three-dimensional object by the division of space into small cubic cells or small parallelepipeds. The size of each cell depends on the local geometric complexity of the object represented [7]. Each cell in space corresponds to a node in the tree and each node is referred to as an octant. To construct an octree, the object is first enclosed by the smallest box that can completely contain the object in any direction. This box (a cube or a parallelepiped) makes up the root level of the octree. It is then subdivided into 8 sub-octants which then represent the first level. The octants are classified into three categories: black (full), white (empty) and grey (partially filled). Black octants are those that are completely contained in the object of interest whereas white ones are those that are completely outside the object. Grey octants are those that are partially inside and outside the object. The subdivision process is performed on grey octants until a desired resolution is reached. The specified accuracy is used to determine the final size of the smallest octants.

Octree decompositions have been used for several years, first in computer graphics [8]. In mechanical engineering, octrees are used for machining simulation [9], for interference detection in five axis machining [10] and in robotics [11]. In rapid prototyping, octree decompositions of 3D models have allowed to realize approximate prototypes before final machining [12].

So the manufacturability analysis will be based on an octree decomposition of the tool CAD model.

\subsection{Manufacturing complexity indexes}

The stages 2 and 5 of the methodology are constituted by manufacturing complexity evaluations. The goal is to determine, at the design stage, which areas of the tool are the most complex-to-manufacture. These are the ones which will impose an increasing manufacturing time, or the ones which will increase overall tool cost or the ones for which it will be difficult to achieve a high quality level.

Two types of manufacturability indexes are defined: local and global. In case of local indexes, a step of decomposition is done according to an octree algorithm. Then the index value is calculated for every grey and black octant, and this value is associated to the fraction of the tool contained in the octant. The higher the index value is, the more difficult-to-manufacture the fraction of the tool contained in the octant is. In order to have a precise view of which area of the tool are the most complex-to-manufacture, a color scale of the index values is created and a color map of manufacturing complexity is displayed. If the decomposition accuracy is not satisfying, another level of the octree decomposition is done, only for grey octants. When a sufficient precision is reached, the decomposition is stopped. The required accuracy must be carefully determined, because if it is too high, it will 
dramatically increase computing time. Nevertheless, it must be not too small with respect to the smallest dimension of the tool. For further examples in this paper, four levels of decomposition have been chosen; it corresponds to an analysis which is neither too wrong nor too computing time expensive. Global indexes are defined for the whole tool. As an example, a simple global index may be calculated from the tool volume. In fact, volume has a great impact on manufacturing time in additive technologies, and the higher the volume is the most complex-to-manufacture the tool is.

The first manufacturability indexes that are developed are presented in Table 1. There are calculated from the geometric parameters of the tool that have a great influence on manufacturing time, cost and quality. These parameters provide information on which tool will be the most difficult-to-manufacture with one manufacturing process (global indexes) and which areas of the tool are the most difficult-to-manufacture (local indexes). Geometric parameters have not the same influence in case of an additive or a subtractive process. That is why some of the following indexes are calculated only for machining or only for layered manufacturing.

Table 1. Manufacturability indexes.

$L X_{\max }, L Y_{\max }$ and $L Z_{\max }$ are the maximal dimensions of the tool in $x, y$ and $z$-direction. $L X_{\operatorname{machine}}, L Y_{\text {machine }}$ and $L Z_{\text {machine }}$ are the lengths of the $X, Y$ and $Z$ axis of the machine. The machines and tool orientation in a machine have to be previously established. For further examples, a Hermle C30U HSM center and an EOS 250 Xtend machine are used with $z$-direction as spindle axis or layer normal orientation.

$C(f)$ index is based on two reports. In most cases, when the milling tool diameter is reduced, machining time increases. Moreover, when the ratio length / diameter of the milling tool increases, the quality of the piece realized is reduced. It corresponds to a diminish of the cutting tool stiffness. $L$ represents the minimal length of the milling tool that can machine the surface contained and is then calculated by the difference of height between the top face of the highest octant and the bottom face of the octant for which $C(f)$ is being calculated. $D$ represents the maximal diameter of the milling tool that can machine the surface contained in the octant, and takes into account both curvature radius of concave surface (a small curvature radius limits the cutting tool diameter that can machine a concave surface) and space between two surfaces, which may also limit cutting tool diameter (Fig. 3). $V$ is the volume of the tool and $S_{\text {ext }}$ is the area of the whole skin surface of the tool.

$x, y$ and $z$ are the coordinates of the gravity centre of the volume of the tool contained in the octant.

$X_{0}, Y_{0}$ and $Z_{0}$ are the coordinates of the bottom face of the octant 0 .

Fig. 3. Example of machining a pocket. $L$ is limited by $L_{\min }, D$ by $r f, r c$ and $D_{\max }$.

At the present time, only manufacturability indexes that are linked to geometric parameters have been implemented in the methodology. Nevertheless, others indexes based on material information (hardness, melting point temperature ...) and technical specification (dimensional and geometric tolerances, surface finish ...) are not presented in this paper.

\subsection{Manufacturability analysis system}

A procedure has been developed to evaluate manufacturing complexity form a tool CAD model. The work has been carried out on a CAD software (SolidWorks 2007) with Visual Basic language. The interface of this system is presented in Fig. 4.

Fig. 4. Interface of manufacturability analysis system.

If the manufacturability index that has been chosen is a global one, the system directly posts its value. The higher the value is, the more difficult-to-manufacture the tool is. In case of a local index, a step of octree decomposition is done. Then the index values are calculated for every grey and black octant. For each octant, the higher the index value is, the more difficult-to-machine the fraction of the tool contained in the octant is. In order to represent the complexity distribution, a map of manufacturing complexity is displayed (with automatic or customized color scale). If the accuracy of the decomposition is not satisfying, another level of octree decomposition is done, only for grey octants (decomposition of black octants do not bring further information). The index values are afresh calculated for new black and grey octants, and a more detailed map of manufacturing complexity is obtained.

For every local indexes $C(i)$, two global values are also calculated:

The maximal value $C(i)_{\max }$;

- $\quad$ A mean value (Eq. (1)), where $V_{j}$ is the volume of the fraction of the tool contained in the octant for which $C(i)_{j}$ is being calculated. 
$C(i)_{\text {mean }}=\frac{\sum_{j}\left(C(i)_{j} \times V_{j}\right)}{\sum_{j} V_{j}}$

This manufacturability analysis system corresponds to the stage 2 and 5 of the methodology. The whole methodology has been applied on test-parts and the next section presents the results to show the possibilities of the hybrid modular tool design methodology.

\section{Examples of using the hybrid modular tool design method}

\subsection{Modular point of view}

The first way of using this method concerns the modular approach, regarding one manufacturing process. The most difficult-to manufacture areas will be improved with a modular point of view, designing modules in order to decrease manufacturability indexes values in these areas.

As an example, a one-piece test-part CAD model is analyzed in term of local and global indexes for machining. Fig. 5 presents the test-part, which is representative of dies and molds traditionally made by High Speed Machining, and Fig. 6 gives the results of the manufacturability analysis for machining process. In case of the $C(f)$ index, which is a local one, a map of manufacturing complexity distribution is displayed and two values are calculated, $C(f)_{\max }$ and $C(f)_{\text {mean }}$.

Fig. 5. One-piece test-part CAD model.

Fig. 6. Second stage of the methodology.

This map provides an accurate view of the machining complexity of the test-part. The easiest-to-manufacture areas are those where there no limitations for the cutting tool diameter $(C(f)=0)$. The most difficult-to manufacture areas are at the bottom of the circular boss, and the surface between the two high bosses because the test-part geometry implies using a long cutting tool with a limited diameter. The next stage of the methodology is to take into account the modular point of view, creating modules manufactured aside and further gathered, to reduce manufacturing complexity in the previous most difficult-to-machine areas, as it can be seen in Fig. 7. In this example, assembly process is not treated.

Fig. 7. Fourth stage of the methodology.

Then manufacturing complexity indexes are calculated for the modules (Table 2), and total indexes are calculated according to Eq. (2). A new map of manufacturing complexity distribution for $C(f)$ index is obtained (Fig. 8).

$C\left(i_{\text {total }}\right)=\frac{\sum_{j} C\left(i_{\text {mod. } j}\right) \times V_{\text {mod. } j}}{\sum_{j} V_{\text {mod. } j}}$

Table 2. Modular test-part manufacturability indexes.

Fig. 8. Fifth stage of the methodology.

The last stage of the methodology is constituted by a comparison of the two manufacturability analyses. So a comparison is done between the manufacturability indexes values obtained at stage 2 and 5 (Fig. 9).

Fig. 9. Manufacturing complexity indexes evolutions between one-piece and modular tools.

It can be seen in the two maps of manufacturing complexity that the modular approach has reduced the machining complexity in the previous most difficult-to-machine areas. Furthermore, the local index values decrease massively whereas the other indexes evolutions are not significant.

So it can be concluded that the modular point of view has allowed designing a modular tool in which machining complexity is reduced. 


\subsection{Hybrid point of view}

Another way of using this system is for comparison of two manufacturing processes (additive and subtractive) for one tool CAD model in order to determine which parts of the tool may advantageously be machined or realized by a layered manufacturing process. This second example is based on quite the same test-part. The test part presented in figure 5 has the following pocket dimensions: $30 \times 50 \times 30 \mathrm{~mm}$, whereas the second one, shown in figure 10 , has a $20 \times 30 \times 30 \mathrm{~mm}$ pocket.

Fig. 10. Second test-part.

Manufacturability indexes are calculated with the manufacturability analysis system for machining (Fig.11).

Fig. 11. Manufacturing complexity evaluation for machining process.

In this second example, it can be seen that changing the dimensions of the test-part provides new very difficult-tomachine areas, according to the flexibility index, at the bottom of the pocket $\left(C(f)_{\max }\right.$ is doubled between the first test-part and the second one). For these areas, a modular approach, similar to the previous example, will not bring a significant decrease in index values. In order to reduce the manufacturing complexity difficulties, an additive process could be used for this test-part realization. So a second manufacturability analysis is done, the values of the manufacturing complexity indexes for layered manufacturing are calculated. The results are presented in Fig. 12.

Fig. 12. Manufacturing complexity evaluation for layered manufacturing.

The two maps of manufacturing complexity distribution, corresponding to the local indexes $C(h)$ and $C(\rho)$, show that some areas of this part are quite difficult-to-manufacture by an additive process.

These analyses indicate that the two manufacturing processes (subtractive and additive) have to be combined in order to produce a hybrid modular tool.

Consequently, the areas which are the most complex-to-machine will advantageously be realized by an additive fabrication technology (Selective Laser Sintering), and the areas that are easy-to-machine will be realized by a machining process (Fig. 13).

Fig. 13. Hybrid modular tool CAD model.

The next stage of the methodology is the manufacturability analysis of the modules. The results are presented in Fig. 14.

Fig. 14. Manufacturing complexity evaluation 2.

The hybrid point of view has allowed creating a hybrid modular tool in which manufacturing complexity is reduced. In fact, the map of machining complexity distribution (Fig. 14(a)) shows that this module is easier-to-machine than the one-piece tool. And the realization of the module 2 by SLS (Fig. 14(b)) does not provide new difficulties of manufacturing with regard to the manufacturability analysis of the one-piece tool. A comparison of the index values between the one-piece tool and the two modules of the hybrid modular one is done on Fig. 15(a) for machining indexes and in Fig. 15(b) for layered manufacturing indexes.

Fig. 15. Comparison of manufacturing indexes.

\section{Conclusion and future work}

This paper presents a new hybrid modular tool design methodology. Starting from the one-piece tool CAD model, global and local manufacturability indexes are calculated. In case of local indexes, which provide a well-detailed view of which areas of the tool are the most complex-to-manufacture, the manufacturability analysis is based on octree decomposition. This new approach allows focusing on the areas of the tool that are the most complex-tomanufacture because an accurate view of the manufacturing complexity distribution is obtained. Then, hybrid and modular points of view help designers to choose between a one-piece design and a hybrid modular one.

In this paper, the manufacturing indexes are based on geometric parameters. It is important to develop indexes that can be calculated at the design stage. Parameters that require a complete manufacturing preparation 
analysis (for example: cutting tool path strategy) are not taken into account to be free from manufacturer skills. Other indexes will be developed, based on material information and technical specifications. Assembly constraints generated by a hybrid modular design have also to be taken into account.

\section{References}

[1] Rivette, M., Mognol, P., Hascoët, J.-Y., 2007, A graph-based methodology for hybrid rapid design, Journal of Engineering Manufacture, 221/4:685-697.

[2] Kerbrat, O., Mognol, P., Hascoët, J.-Y., 2010, Manufacturability analysis to combine additive and subtractive processes, Rapid Prototyping Journal, 16/1:63-72.

[3] Gupta, S.K., Das, D., Regli, W.C., Nau, D.S., 1997, Automated manufacturability analysis: A survey, Research in Engineering Design 9/3:168-190.

[4] Newman, S.T., Nassehi, A., Xu, X.W., Rosso Jr., R.S.U., Wang, L., Yusof, Y., Ali, L., Liu, R., Zheng, L.Y., Kumar, S., Vichare, P., Dhokia, V., 2008, Strategic advantages of interoperability for global manufacturing using CNC technology, Robotics and Computer-Integrated Manufacturing, 24:699-708.

[5] Bonnard, R., Mognol, P., Hascoët, J.-Y., 2008, Rapid prototyping project description in STEP-NC model, Proceedings of the $6^{\text {th }}$ CIRP International Conference on Computation in Manufacturing Engineering (Naples, Italy).

[6] Meagher, D., 1982, Geometric modeling using octree encoding, Computer graphics and image processing, 19:129-147.

[7] Kim, J., 1998, NC verification using octree, M.Sc in Mechanical Engineering, MIT.

[8] Szeliski, R., 1990, Real-time octree generation from rotating object, Cambridge Research Laboratory Technical Report Series.

[9] Kim, Y.-H., Ko, S.-L., 2006, Improvement of cutting simulation using the octree model, International Journal of Advanced Manufacturing Technology, 28:1152-1160.

[10] Ding, S., Manna, M.A., Poo, A.N., 2004, Oriented bounding box and octree based global interference detection in 5-axis machining of free-form surfaces, Computer-Aided Design, 36:1281-1294.

[11] Wenger, P. Chablat, D., 1998, Workspace and assembly modes in fully-parallel robots: a descriptive study, Proceedings of the $6^{\text {th }}$ International Symposium on Advances in Robot Kinematics (Salzburg, Austria)..

[12] Medellin H., Corney, J., Davies, J.B.C., Lim, T., Ritchie, J.M., 2006, Algorithms for the physical rendering and assembly octree models, Computer-Aided Design, 38:69-85. 
Figure 1

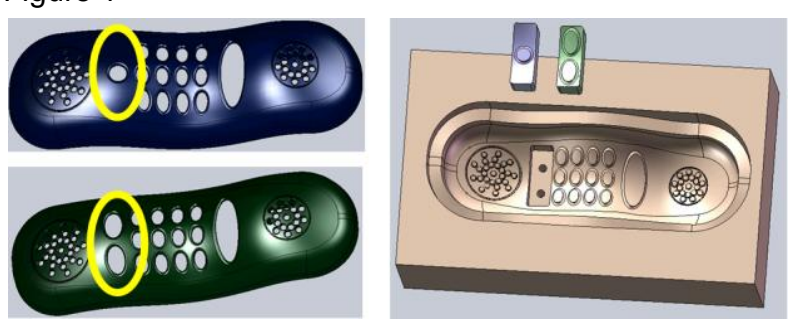

Figure 2

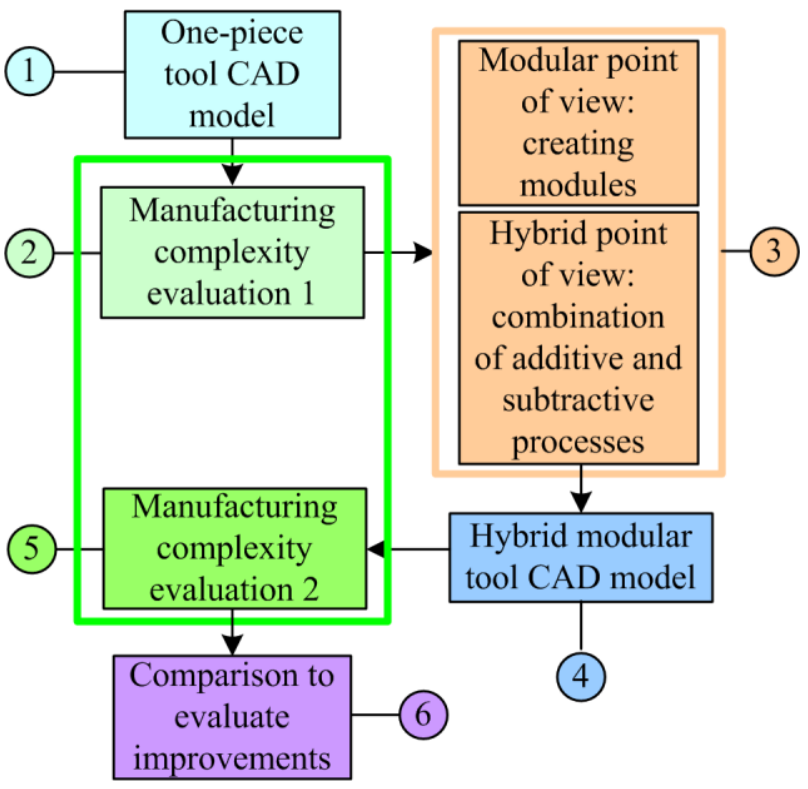

Figure 3

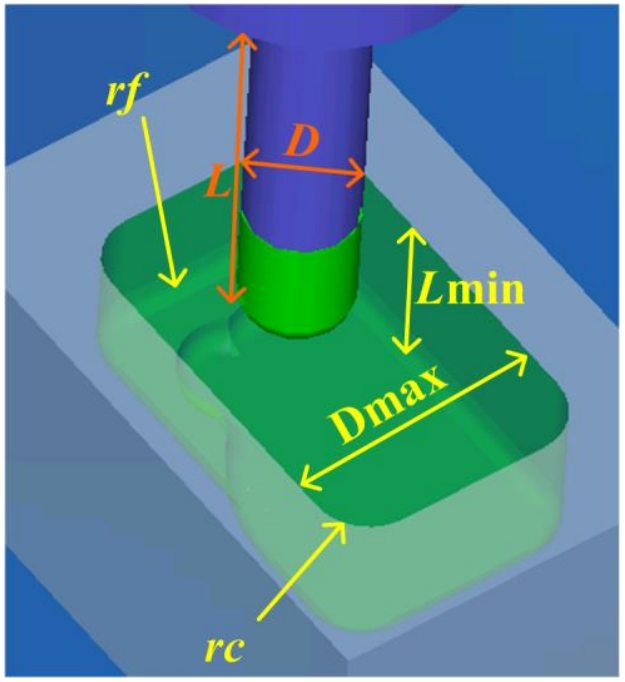


Figure 4

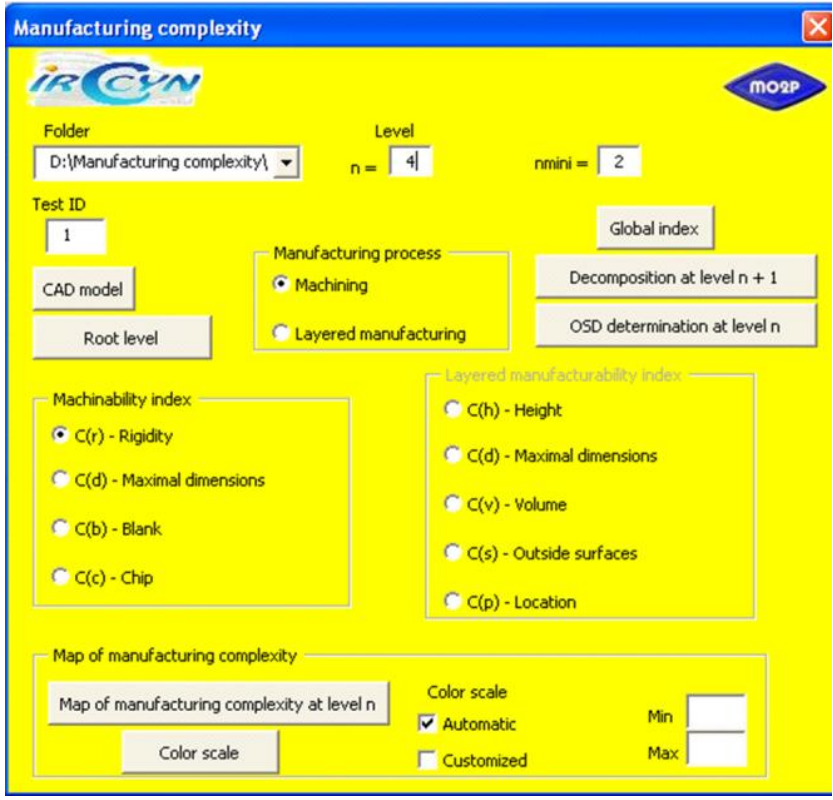

Figure 5
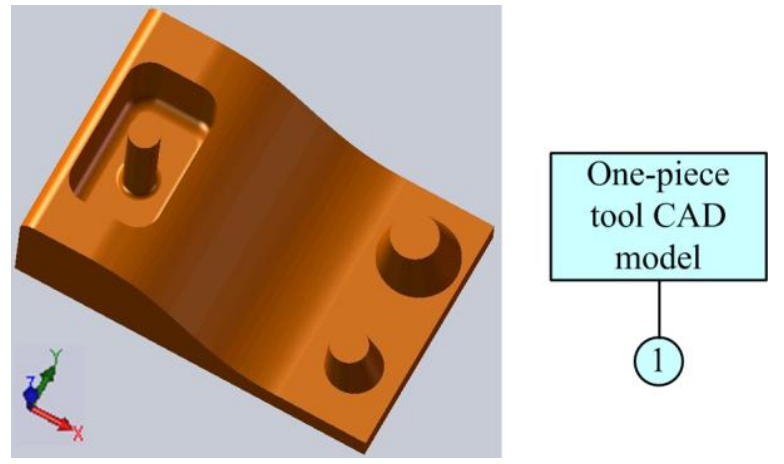

\section{Figure 6}

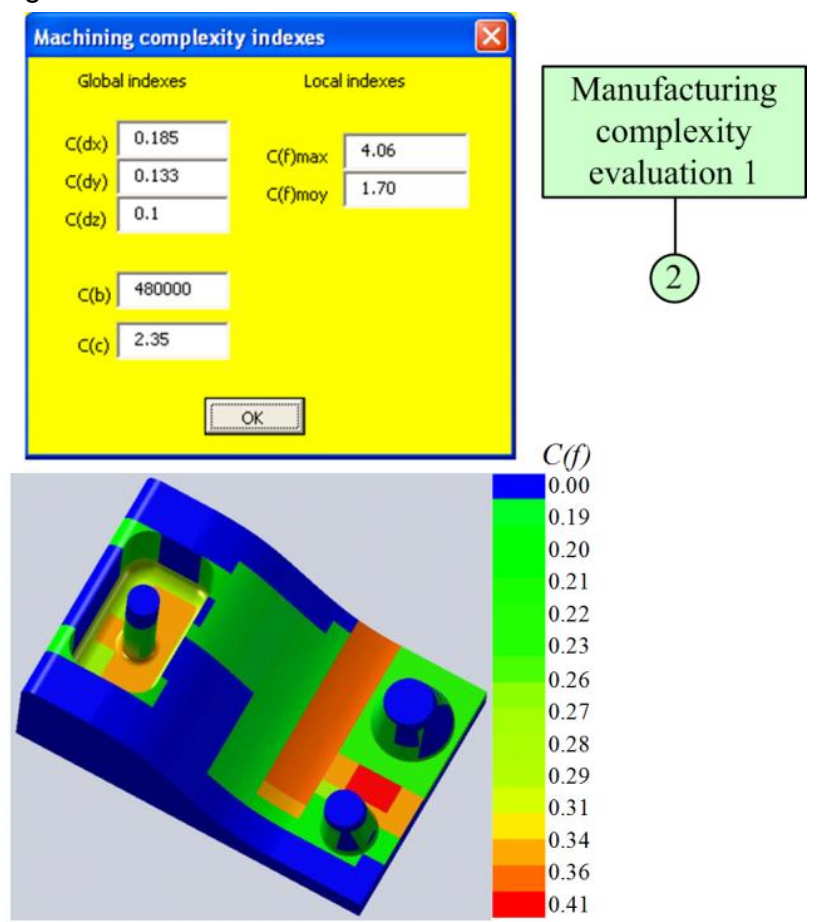


Figure 7

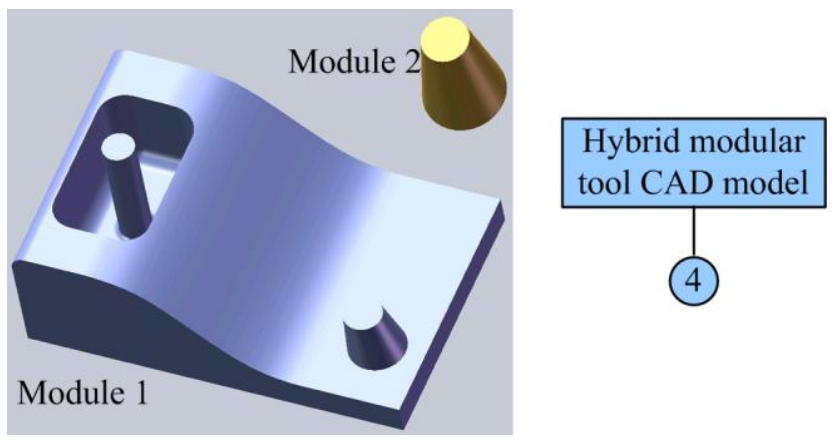

Figure 8

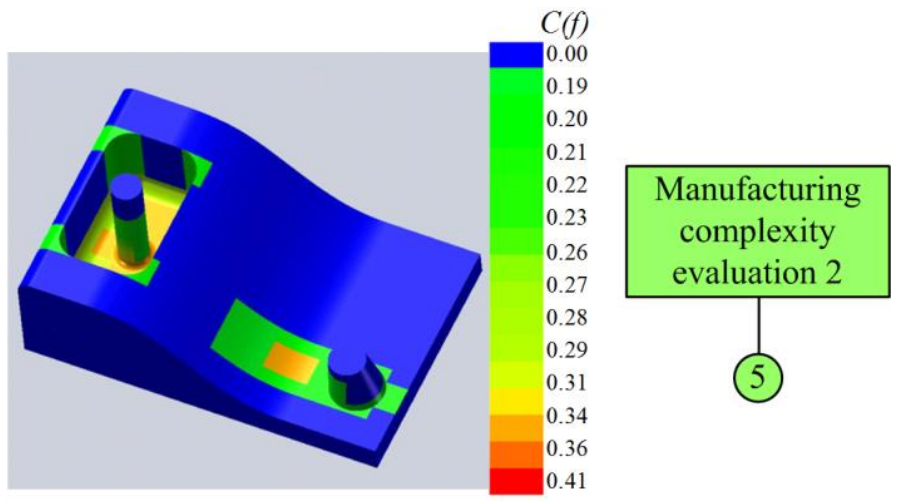

Figure 9

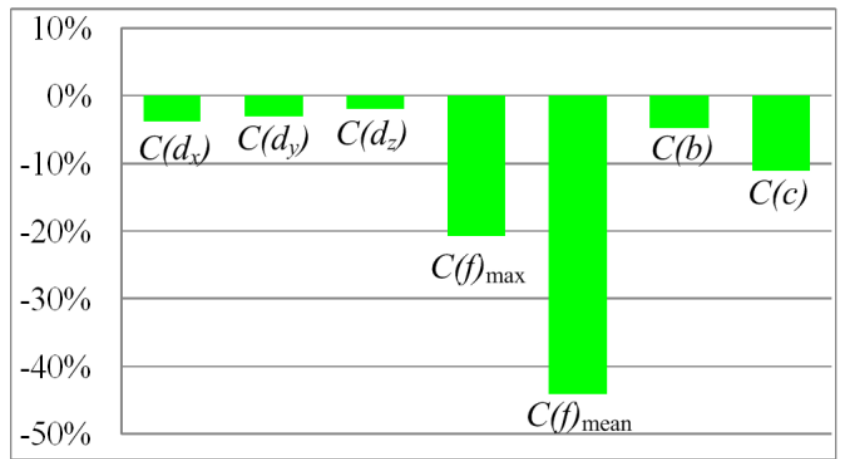

Figure 10

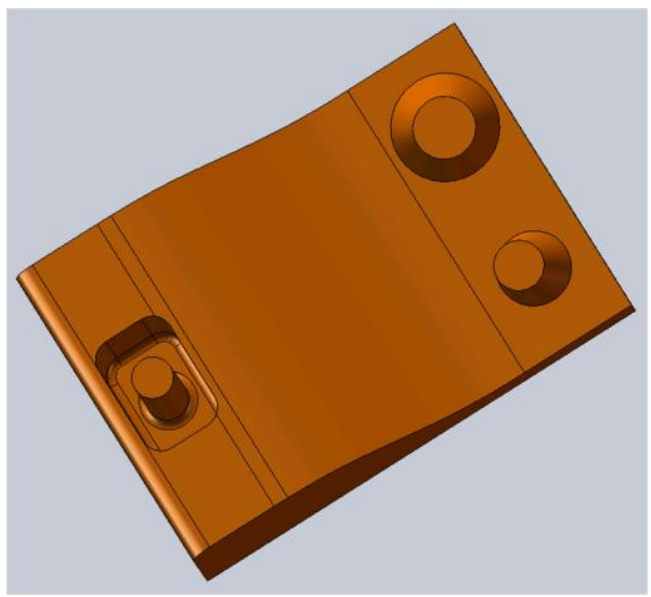


Figure 11

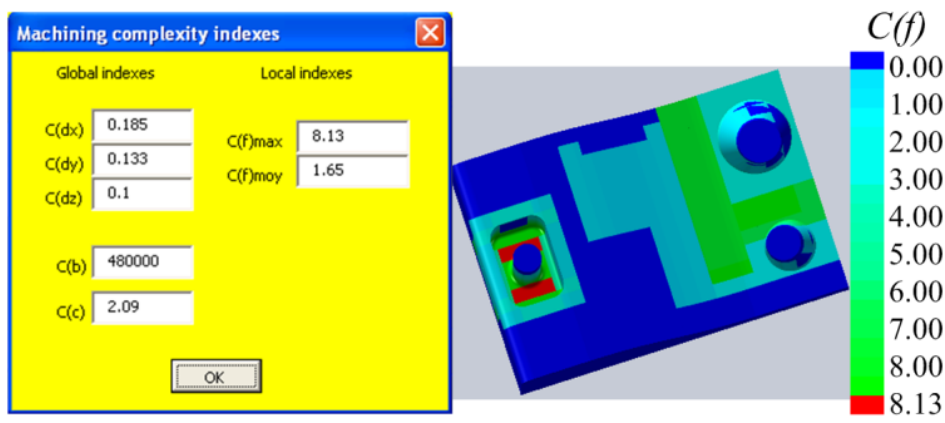

Figure 12

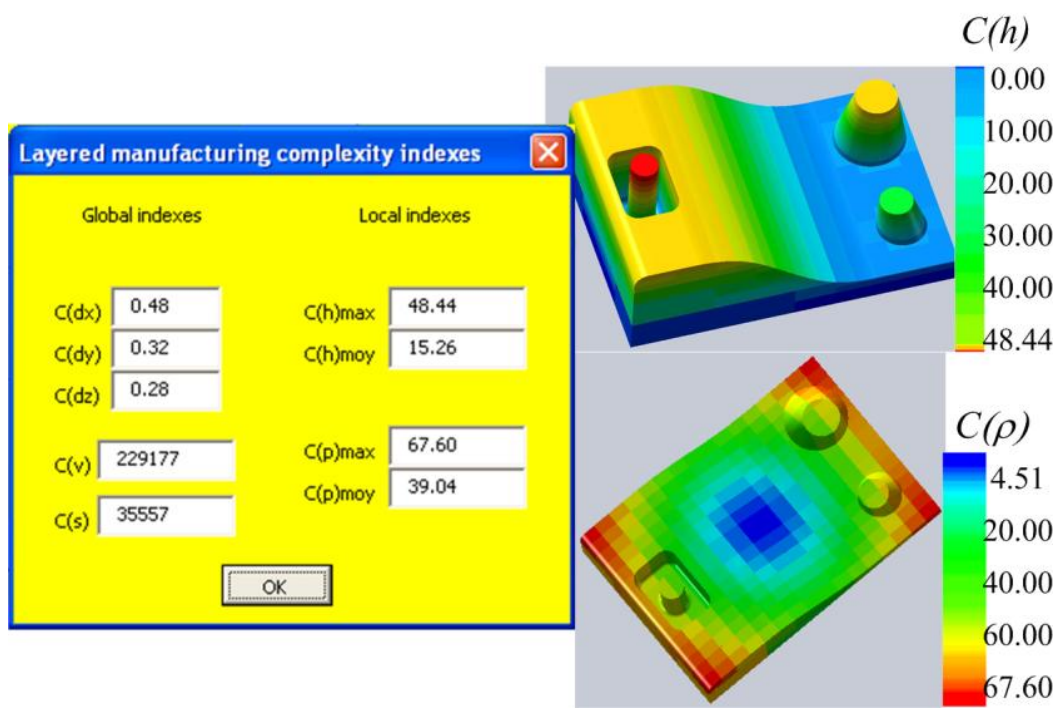

Figure 13

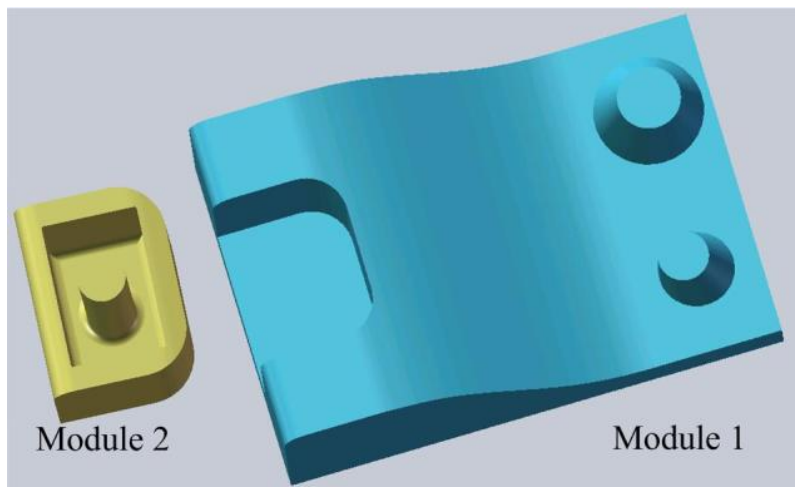


Figure 14

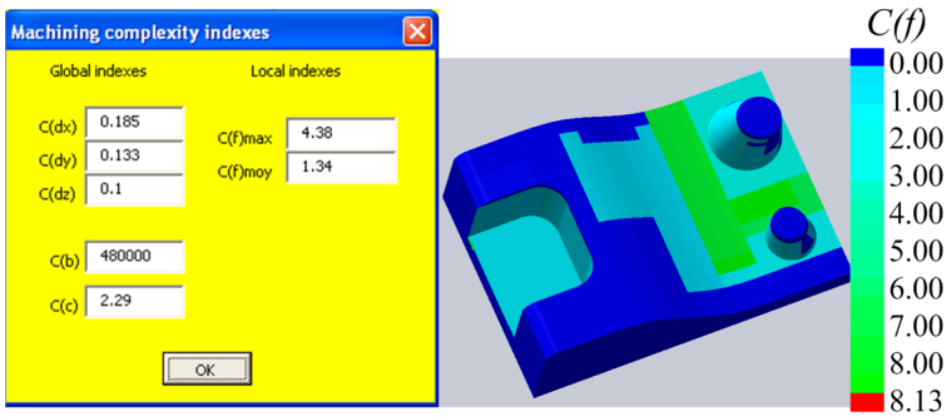

(a) Manufacturability analysis for module 1, realized by HSM

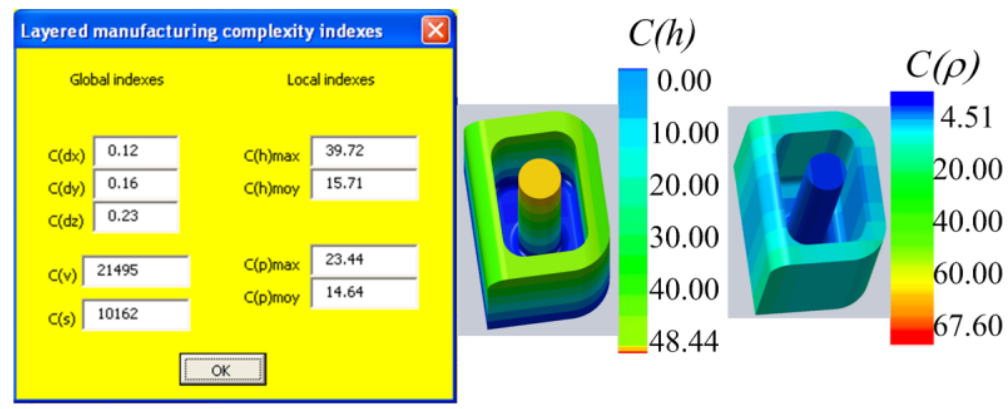

(b) Manufacturability analysis for module 2, realized by SLS

Figure 15

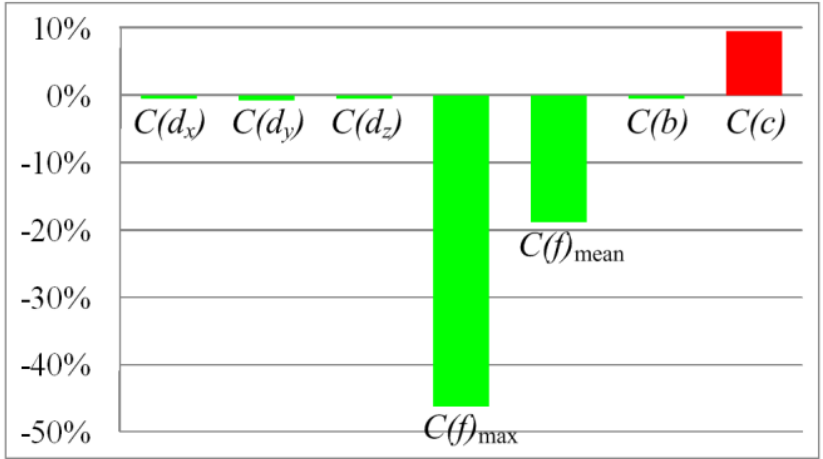

(a) Comparison for machining indexes

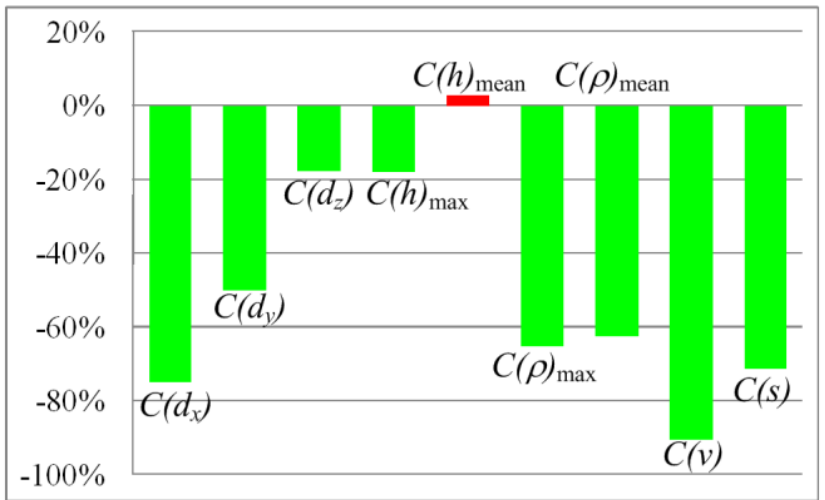

(b) Comparison for layered manufacturing indexes 


\begin{tabular}{|c|c|c|c|c|}
\hline Index & Linked to & Type & Applied to & Value \\
\hline$C\left(d_{x}\right)$ & $\begin{array}{c}\text { Maximal } \\
\text { dimension in } x \text { - } \\
\text { axis }\end{array}$ & Global & $\begin{array}{l}\text { Machining / } \\
\text { Layered } \\
\text { manufacturing }\end{array}$ & $C\left(d_{x}\right)=\frac{L X_{\max }}{L X_{\text {machine }}}$ \\
\hline$C\left(d_{y}\right)$ & $\begin{array}{c}\text { Maximal } \\
\text { dimension in } y \text { - } \\
\text { axis }\end{array}$ & Global & $\begin{array}{l}\text { Machining / } \\
\text { Layered } \\
\text { manufacturing }\end{array}$ & $C\left(d_{y}\right)=\frac{L Y_{\max }}{L Y_{\text {machine }}}$ \\
\hline$C\left(d_{z}\right)$ & $\begin{array}{c}\text { Maximal } \\
\text { dimension in } z- \\
\text { axis }\end{array}$ & Global & $\begin{array}{l}\text { Machining / } \\
\text { Layered } \\
\text { manufacturing }\end{array}$ & $C\left(d_{z}\right)=\frac{L Z_{\max }}{L Z_{\text {machine }}}$ \\
\hline$C(f)$ & $\begin{array}{l}\text { Cutting tool } \\
\text { flexibility }\end{array}$ & Local & Machining & $C(f)=\frac{L}{D}$ \\
\hline$C(b)$ & Blank volume & Global & Machining & $C(b)=L X_{\text {max }} \times L Y_{\text {max }} \times L Z_{\text {max }}$ \\
\hline$C(c)$ & Quantity of chips & Global & Machining & $C(c)=\frac{L X_{\max } \times L Y_{\max } \times L Z_{\max }}{V}$ \\
\hline$C(v)$ & Volume & Global & $\begin{array}{c}\text { Layered } \\
\text { manufacturing }\end{array}$ & $C(v)=V$ \\
\hline$C(h)$ & Height & Local & $\begin{array}{c}\text { Layered } \\
\text { manufacturing }\end{array}$ & $C(h)=z-Z_{0}$ \\
\hline$C(s)$ & Skin surface & Global & $\begin{array}{c}\text { Layered } \\
\text { manufacturing }\end{array}$ & $C(s)=S_{e x t}$ \\
\hline$C(\rho)$ & $\begin{array}{l}\text { Distance from the } \\
\text { centre of the } \\
\text { platform }\end{array}$ & Local & $\begin{array}{c}\text { Layered } \\
\text { manufacturing }\end{array}$ & $C(\rho)=\sqrt{\left(x-X_{0}\right)^{2}+\left(y-Y_{0}\right)^{2}}$ \\
\hline
\end{tabular}

Table 2

\begin{tabular}{|c|c|c|c|}
\hline Indexes & Module 1 & Module 2 & Total \\
\hline$C\left(d_{x}\right)$ & 0.185 & 0.043 & $\mathbf{0 . 1 7 8}$ \\
\hline$C\left(d_{y}\right)$ & 0.133 & 0.043 & $\mathbf{0 . 1 2 9}$ \\
\hline$C\left(d_{z}\right)$ & 0.100 & 0.060 & $\mathbf{0 . 0 9 8}$ \\
\hline$C(f)_{\text {max }}$ & 3.39 & 0 & $\mathbf{3 . 2 2}$ \\
$C(f)_{\text {mean }}$ & 0.99 & 0 & $\mathbf{0 . 9 5}$ \\
\hline$C(b)$ & 480000 & 20280 & $\mathbf{4 5 7} 273$ \\
\hline$C(c)$ & 2.09 & 2.01 & $\mathbf{2 . 0 9}$ \\
\hline
\end{tabular}

